\title{
Experimental Airborne Transmission of Porcine Postweaning Multisystemic Wasting Syndrome
}

\author{
C. S. Kristensen, ${ }^{1}$ C. K. Hjulsager, ${ }^{2}$ K. Vestergaard, ${ }^{1}$ K. Dupont, ${ }^{2}$ V. Bille-Hansen, ${ }^{2}$ C. Enøe, ${ }^{2}$ \\ S. E. Jorsal, ${ }^{2}$ P. Bakbo, ${ }^{1}$ and L. E. Larsen ${ }^{2}$ \\ ${ }^{1}$ Pig Research Centre, Danish Agriculture \& Food Council, Vinkelvej 11, 8620 Kjellerup, Denmark \\ ${ }^{2}$ National Veterinary Institute, Technical University of Denmark, Bülowsvej 27, 1870 Frederiksberg C, Denmark \\ Correspondence should be addressed to L. E. Larsen; lael@vet.dtu.dk
}

Received 31 July 2012; Accepted 6 January 2013

Academic Editor: Slobodan Paessler

Copyright (C) 2013 C. S. Kristensen et al. This is an open access article distributed under the Creative Commons Attribution License, which permits unrestricted use, distribution, and reproduction in any medium, provided the original work is properly cited.

\begin{abstract}
The objective of these studies was to investigate if porcine postweaning multisystemic wasting syndrome (PMWS) could be induced in healthy pigs following contact with air from pigs with clinical signs of PMWS. The pigs were housed in different units. Either 31 (study I) or 25 (study II) pigs with clinical symptoms of PMWS from a PMWS-affected herd and 25 healthy pigs from a PMWS-free, but PCV2-positive, herd were housed in unit A. Fifty pigs from a PMWS-free herd were housed in unit B, which were connected by pipes to unit A. In unit C, 30 pigs from a PMWS-free herd were housed as controls. In study II, the pigs in units A and B from the PMWS-free herd developed clinical signs of PMWS 2-3 weeks after arrival. PMWS was confirmed at necropsy and the diseased pigs had increased PCV2 load and increased antibody titers against PCV2 in serum that coincided with the development of clinical signs typical of PMWS. Sequence analysis revealed that the PCV2 isolate belonged to genotype $2 \mathrm{~b}$. In conclusion, the present study showed that PMWS can be induced in pigs from a PMWS-free herd by airborne contact with pigs from a PMWS-affected herd.
\end{abstract}

\section{Introduction}

Postweaning multisystemic wasting syndrome (PMWS) is an important disease in weaned pigs worldwide. PMWS was first described in Canada in 1991 as a chronic disease with progressive weight loss in pigs from 4-16 weeks of age [1]. Since then, the disease has been diagnosed in many countries in North America, Asia, and Europe including Denmark [2, 3]. The clinical signs of PMWS comprise unthriftiness/wasting, paleness of the skin, enlarged lymph nodes, and occasionally jaundice, respiratory symptoms, or diarrhoea $[1,3,4]$. Affected pigs have lesions in lymphoid organs characterized by lymphoid depletion and the presence of giant cells and inclusion bodies [4-7]. PCV2 has proved to be necessary but not sufficient for development of PMWS, since the virus is present in both affected and PMWS-free pigs and herds $[4,8]$.

The PCV2 virus is transmitted between pigs by the orofecal and/or respiratory routes $[9,10]$ and vertical transmission has also been documented $[4,11]$. The high prevalence of PCV2 in almost all herds of all pig-producing countries indicates that the transmission of PCV2 is very effective [1215]. In contrast, only a few studies have been performed on the "transmission" of the PCV2-associated disease complexes (PCVDs), that is, whether PMWS can be "transmitted" from PMWS-affected to PMWS-free pigs. A study performed in New Zealand demonstrated disease development in healthy pigs in direct or indirect contact with PMWS-affected pigs when they were mingled at 4 weeks of age but not when they were mingled at 12 weeks of age [16]. Spatial analysis carried out in Denmark and Great Britain concluded the existence of significant spatiotemporal clusters, suggesting the spread of an infectious agent from farm to farm [17, 18]. Descriptive epidemiology in Sweden also showed a clear tendency of the epidemic to move slowly from south to north [19].

Previously we have shown that PMWS can be transmitted from pig to pig by close contact [20] and PCV2 has been found in air samples collected in PCV2-positive herds [21], but it still remained unclear if PMWS can be transmitted through air. The purpose of the present studies was to 
examine the possibility of airborne transmission of PMWS in a controlled semiexperimental setup.

\section{Materials and Methods}

2.1. Air Transmission Model. Two studies were performed. For the studies, three containers were constructed as "pig units" (unit A, unit B, and unit C). Units A and B were placed one meter apart and connected by pipes (Tables $2-4$ ). In unit A, air pressure was increased by a ventilator mounted in the gable that blew fresh air into the room through four adjustable valves. Exhaust air was pushed out through a stack in the roof. In unit $\mathrm{B}$, air pressure was decreased by a ventilator mounted in a stack in the roof that controlled exhaust air to the outside. Air was sucked into the room through four valves. Thus, air pressure in unit A was always higher than the air pressure in unit $B$, resulting in air transfer from unit A to unit B through the pipes. The volume of air transferred through the pipes depended on the number and diameter of the pipes as well as the pressure difference between the units. Thus, to maintain a particular rate of air transfer, the pipes diameter could be adjusted with orifices. The ventilation system, the opening valves, and the pipes were all calibrated before the beginning of the studies. Air pressure differences were measured every $10 \mathrm{~min}$ in order to calculate the amount of air transferred. The amount of air transferred from unit A to unit B, expressed as percent of ventilation intake, was on average $83 \%$ (S.D. 27\%) for study I and 69\% (S.D. 27\%) for study II.

Units A and B were $2.5 \times 9.5$ meter each and consisted of two rooms. Personnel entered through the first room $(2.5 \times$ 2.5 meter) and changed clothes there. The pigs were housed in the second room which was $17.5 \mathrm{~m}^{2}$ and had slatted plastic floor. Each unit had a two-climate system with coverings and straw bedding. Units A and B were placed at research facility $1,2.2 \mathrm{~km}$ from other pig herds.

Unit $\mathrm{C}$ was placed at research facility 2 approximately $3.1 \mathrm{~km}$ from unit $\mathrm{A}$ and unit $\mathrm{B}$, with no pigs within a range of $2.3 \mathrm{~km}$ from the unit. The unit was $18 \mathrm{~m}^{2}$ and consisted of one room with slatted plastic floor and a two-climate system with coverings and straw bedding.

2.2. Study Setup. The pigs (Danish Landrace/Duroc crossbred), 8-12 weeks of age, were obtained from three different herds that were all PCV2 infected and PCV2 unvaccinated, verified by serological reactions. Two of the herds were PMWS affected (PMWS-1 and PMWS-2) and one was PMWS-free (PMWS-free). The PMWS diagnosis within the PMWS-affected herds were based on high prevalence of unthrifty pigs, high mortality among weaners (5-6\% and $15 \%$ ), and a positive histopathological examination of lymphoid tissue together with detection of PCV2 antigens according to the EU definition (http://www.pcvd.org/). The PMWS-free herd was characterized by low mortality among weaners (2-3\%). This status persisted during the study period and until three months after.

All three herds were infected with Mycoplasma hyopneumoniae and Actinobacillus pleuropneumoniae serotype 6. The PMWS-1 and the PMWS-free herds were infected with
PRRSV-EU and PMWS-2 with A. pleuropneumoniae serotype 2, toxigenic Pasteurella multocida, and PRRSV-US. A vaccine against porcine parvovirus was used in sows in all three herds. A vaccine against toxigenic $P$. multocida was used in herd PMWS-1.

The PMWS-affected herds were visited by the veterinarian 3-4 days before the start of each of the studies at which time 25 ( 31 for study II) pigs with clinical symptoms of PMWS among weaners were selected and ear tagged. The same veterinarian visited the PMWS-free herd one week before the study and started to make sure that no clinical signs of PMWS were present. From the PMWS-free herd, 105 pigs were transferred to the research facilities at weaning in each study.

The pigs from the PMWS-nonaffected herd were randomly assigned to unit A, unit B, and unit C. Approximately half of the pigs in unit $A$ and all the pigs in unit $B$ and $C$ originated from the PMWS-free herd in both studies. The remaining pigs in unit A originated from the PMWS-affected herd PMWS-1 in study I and PMWS-2 in study II (Table 1).

On the day of arrival (day 1), all the pigs were marked with individual ear tags and weighed.

Water and feed without antibiotics were offered ad libitum throughout the study period. To prevent diarrhoea $2500 \mathrm{ppm}$ zinc oxide was used in the feed the first 10 days after arrival in both studies.

Transmission of pathogens by personnel was prevented through biosecurity measures (changing clothes before entering units, using disposable gloves and masks covering hair, nose, and mouth). A twelve-hour pig contact quarantine was established before entering the units, within which a shower had to be taken and clothes changed. The pigs were handled in the same order every day: first unit C, then unit B, and finally unit A. Transmission by insects was prevented by a fly net at the air inlet in unit B. The duration of both studies was 69 days.

2.3. Clinical Observation and Postmortem Examination. The pigs were monitored for clinical signs of PMWS three times weekly by the veterinarian.

All the pigs that demonstrated severe clinical disease were euthanized during the study. At the end of the study, all the unthrifty pigs were euthanized. All the pigs that were euthanized or died spontaneously were necropsied.

During necropsy, a tissue sample was taken from the inguinal lymph node, the mesenterical lymph nodes, and the spleen and fixed by immersion into $4 \%$ paraformaldehyde at $22^{\circ} \mathrm{C}$ for histopathological examination. Sections of paraffinembedded paraformaldehyde-fixed tissue were stained with hematoxylin and eosin for histomorphological evaluation and immunohistochemistry for PCV2 by specific monoclonal antibodies as previously described [22]. The individual pigs were diagnosed with PMWS if they showed clinical signs together with characteristic histopathological lesions in lymphoid tissue (lymphocyte depletion together with histiocytic infiltration and/or giant cells and/or inclusion bodies) and detection of moderate or massive amounts of PCV2 antigen by immunofluorescence. This is in accordance with the EU definition (http://www.pcvd.org/). 
TABLE 1: Distribution of pigs in the two experiments.

\begin{tabular}{|c|c|c|c|c|}
\hline & \multicolumn{3}{|c|}{ Facility 1} & \multirow{2}{*}{$\begin{array}{c}\text { Facility } 2 \\
\text { Unit C }\end{array}$} \\
\hline & & & Unit B & \\
\hline \multicolumn{5}{|l|}{ Experiment 1} \\
\hline Status herd of origin & PMWS-1 & PMWS-free & PMWS free & PMWS free \\
\hline Number of pigs & 25 & 25 & 50 & 30 \\
\hline Mean weight $(\mathrm{kg})$ & 14.2 & 11.7 & 11.0 & 11.3 \\
\hline \multicolumn{5}{|l|}{ Experiment 2} \\
\hline Status herd of origin & PMWS-2 & PMWS free & PMWS free & PMWS free \\
\hline Number of pigs & 31 & 25 & 50 & 30 \\
\hline Mean weight (kg) & 14.3 & 9.3 & 9.3 & 9.0 \\
\hline
\end{tabular}

2.4. Blood Sampling and Serological Analysis. Blood samples were collected from all the pigs at the beginning of the studies. Additional blood samples were collected from the pigs before euthanization or at termination of the study. Blood was rescued from the heart of dead pigs when possible.

The serum samples were tested for PRRSV antibodies using immunoperoxidase monolayer assay (IPMA) as previously described [23]. The IPMA was carried out as a double test [24] using MARC-145 cells infected with a Danish field strain of PRRSV and with an American vaccine strain ("Ingelvac" PRRS MLV, Boehringer Ingelheim), respectively. Antibodies against PCV-2 were measured by an in-house developed ELISA [20]. Tests for antibodies against PCV2, PRRS (Types I and II), and PCV2 quantification were only carried out on samples from study II.

The geometric mean was calculated for each group and sampling in study II as the exponential of the arithmetic mean of the log-transformed titer values $(>0)$ (Table 4$)$. The proportion of samples with a titer value above 0 was also determined (Table 4).

2.5. Real-Time PCR and Sequencing. In study II, PCV2 was quantified by real-time PCR on serum samples from selected pigs from the PMWS-affected herd in unit A and from all pigs in unit B at arrival and, when blood was rescued, at death or at termination of the study. Real-time PCR was performed as previously described [25]. The PCV2 genome of selected samples was PCR amplified in three overlapping reactions and sequenced as previously described $[15,26]$.

\section{Results}

3.1. Clinical Signs and Postmortem Examination. The mean weight of the pigs at arrival is listed in Table 1 . In study I, one of the pigs in unit B died on day 2 after the beginning of the study due to diarrhea and one of the pigs was euthanized due to lameness four weeks after the beginning of the study. No other clinical signs were seen among the pigs from the PMWS-free herd in the three units. Fourteen of the pigs from the PMWSaffected herd in unit A were necropsied. Nine of these pigs were diagnosed with PMWS according to the EU definition (Table 2).

In study II, the pigs from the PMWS-free herd in unit A started to show clinical signs of PMWS two weeks after mingling with the pigs from the PMWS-affected herd. In unit $B$, receiving air from unit $A$, the clinical signs were seen three weeks after the start of the study. The most prominent clinical signs were depression, unthriftiness, and wasting. Some of the pigs had dyspnea or diarrhoea. At necropsy the predominant findings were enteritis followed by heavy lungs and bronchopneumonia. Many pigs had enlarged bronchial and inguinal lymph nodes and some had enlarged mesenteric lymph nodes (Table 2). Fourteen of the pigs from the PMWSaffected herds in unit A were necropsied, and three of these were diagnosed with PMWS. Ten of the pigs from the PMWSfree herd in unit A were necropsied, and three of these were diagnosed with PMWS (Table 3). Of the pigs in unit B, 20 were necropsied and 13 of these were diagnosed with PMWS. In both units approximately one-third of the pigs died or were euthanized due to evident wasting. None of the pigs in unit $\mathrm{C}$ showed any signs of wasting or any other clinical symptoms of disease. Eight of the healthy pigs from unit $\mathrm{C}$ were euthanized and necropsied and showed no signs of PMWS.

3.2. Serological Analysis. The blood samples from study I were not analyzed due to lack of clinical signs and confirmed PMWS diagnosis in pigs from the PMWS-free herd.

The serological profiles of the different groups at arrival and at termination of study II are shown in Table 4 . The pigs from both the PMWS-free and the PMWS-affected herds had antibodies against PCV2 at arrival. The levels of antibodies in the pigs from the PMWS-affected herd were significantly higher than the levels in the pigs from the PMWS-free herd. A marked increase in the level of PCV2 antibodies was seen in all the pigs in units $\mathrm{A}$ and $\mathrm{B}$ in contrast to a decrease in the level of PCV2 antibodies seen in the pigs in unit $\mathrm{C}$ at facility 2.

The pigs from the PMWS-affected herd had high levels of antibodies against PRRSV Type II (US) at arrival and the level remained high until the end of the study. The pigs from the PMWS-free herd had no antibodies against PRRSV Type II at arrival. Pigs from this herd placed in units A and B showed a marked increase in the level of antibodies towards PRRSV Type II from the start to the end of the study in contrast to pigs placed in unit $\mathrm{C}$ at facility 2 which did not develop antibodies against PRRSV (Table 4). Apart from the low level in some pigs, all pigs were free from antibodies to PRRSV Type I (EU) at arrival. 
TABLE 2: Gross lesions detected on autopsied pigs from the four units in experiment 2.

\begin{tabular}{lcccc}
\hline Gross lesions & Unit A & Unit B & Unit C \\
PMWS-2 & PMWS-free & 10 & 20 & 8 \\
Number of pigs autopsied & 14 & 4 & 15 & 0 \\
Heavy lungs & 5 & 3 & 8 & 0 \\
Bronchopneumonia & 5 & 9 & 16 & 0 \\
Enteritis/dilated intestines & 12 & 6 & 4 & 0 \\
Enlarged mesenterical lnn. & 5 & 6 & 10 & 0 \\
Enlarged bronchial lnn. & 9 & 6 & 11 & 0 \\
Enlarged inguinal lnn. & 6 & & & 0 \\
\hline
\end{tabular}

TABLE 3: Number of pigs diagnosed with PMWS.

\begin{tabular}{|c|c|c|c|c|}
\hline \multirow{2}{*}{ Status herd of origin } & \multicolumn{2}{|c|}{ Unit A } & \multirow{2}{*}{$\begin{array}{c}\text { Unit B } \\
\text { PMWS-free }\end{array}$} & \multirow{2}{*}{$\begin{array}{c}\text { Unit C } \\
\text { PMWS-free }\end{array}$} \\
\hline & PMWS pos. & PMWS-free & & \\
\hline \multicolumn{5}{|l|}{ Experiment 1} \\
\hline Number euthanized & 14 & 0 & $2^{1}$ & 0 \\
\hline PMWS positive & 9 & 0 & 0 & 0 \\
\hline \multicolumn{5}{|l|}{ Experiment 2} \\
\hline Number euthanized & 14 & 10 & 20 & 8 \\
\hline PMWS positive & 3 & 3 & 13 & 0 \\
\hline
\end{tabular}

${ }^{1}$ One euthanized due to diarrhea, another due to lameness.

3.3. PCV2 Levels in Serum and Sequencing. The blood samples from study I were not analyzed due to lack of clinical signs and confirmed PMWS diagnosis in pigs from the PMWS-free herd.

In study II, from unit A 11 of the pigs from the PMWSaffected herd that were necropsied were tested by quantitative PCR for PCV2 at the start of the experiment. The three pigs that got a confirmed PMWS diagnosis had PCV2 titers of $1,5 \mathrm{E}+10 ; 8,7 \mathrm{E}+10$; and $6,5 \mathrm{E}+07$, respectively, at the start of the experiment (data not shown). Blood was only rescued from one of these pigs at necropsy showing 4,0E +11 copies of PCV2 pr. mL serum. The PMWS diagnosis could not be confirmed for the remaining eight pigs and these pigs had PCV2 titers at 7, 3E +07 copies of PCV2 pr. mL serum or lower at necropsy, which was slightly lower than at the start of the experiment.

From unit B, 38 pigs were tested for PCV2 DNA by quantitative PCR at arrival and at necropsy or at termination of the study (Figure 1). At the start of the experiment, PCV2 was either undetectable (35 pigs) or very low (three pigs). At the end of the experiment, they all developed high PCV2 titers ranging from $2,8 \mathrm{E}+04$ to $6,4 \mathrm{E}+10$ copies of PCV2 pr. $\mathrm{mL}$. Eight of the pigs had more than $6,7 \mathrm{E}+07$ copies of PCV2 pr. $\mathrm{mL}$ serum at necropsy and they were all confirmed PMWS cases by histopathological examination. Additionally four of the pigs analyzed with quantitative PCR were examined histologically due to clinical signs of PMWS but did not fulfil the histopathological criteria for PMWS diagnosis and they had lower PCV2 titers in the sera (from 3, 9E +04 to $4,1 \mathrm{E}+06$ copies pr. $\mathrm{mL}$ ) (Figure 1 ).

PCV2 rescued from serum of six pigs from the PMWSaffected herd in unit $\mathrm{A}$ at arrival and from tissue collected at necropsy from two of the pigs from the PMWS-free

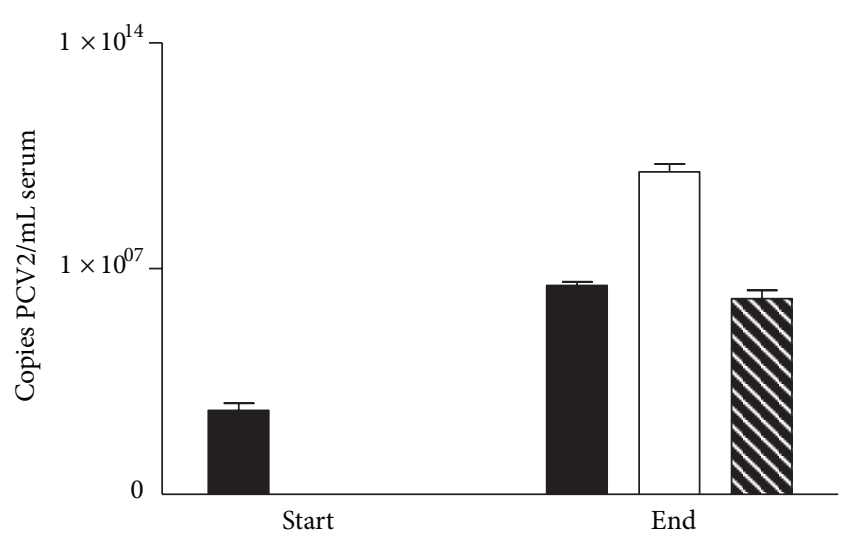

FIgURE 1: Level of PCV2 in serum (copies per mL of serum) of pigs from PMWS-free herds in unit B (study II) at arrival and at termination of the study. Pigs showing no signs of PMWS (black); pigs with confirmed PMWS (white) and pigs showing signs of PMWS, but which did not fulfil the criteria at necropsy (black and white).

herd which developed PMWS in unit B was sequenced. All sequenced isolates belonged to the genotype $2 \mathrm{~b}$ and were closely related to circulating Danish PCV2 isolates [26] (data not shown). The positions of the few base differences identified in PCV2 genomes isolated from the pigs from units $A$ and $B$ are shown in Table 5. Two variants of PCV2 were isolated from pigs from the PMWS-affected herd in unit A on arrival. One of these variants was also found in both of the pigs that developed PMWS in unit B. 
TABLE 4: Geometric mean of positive antibody titers (proportion of positive samples) against PRRSV EU, PRRSV US, and PCV2, at arrival and at the end of study II.

\begin{tabular}{|c|c|c|c|c|}
\hline \multirow{3}{*}{$\begin{array}{l}\text { Facility } \\
\text { Unit } \\
\text { Status of herd }\end{array}$} & \multicolumn{3}{|c|}{1} & \multirow{3}{*}{$\begin{array}{c}2 \\
\text { C } \\
\text { PMWS-free }\end{array}$} \\
\hline & \multicolumn{2}{|c|}{ A } & B & \\
\hline & PMWS affected & PMWS-free & PMWS-free & \\
\hline \multicolumn{5}{|l|}{ PRRS EU } \\
\hline Arrival & $79.2(P=7 / 31)$ & $50.0(P=2 / 25)$ & $50.0(P=9 / 50)$ & $50.0(P=2 / 30)$ \\
\hline End & $135.4(P=21 / 26)$ & $123.7(P=16 / 23)$ & $146.2(P=27 / 44)$ & n.a. ${ }^{*}(P=0 / 30)$ \\
\hline \multicolumn{5}{|l|}{ PRRS US } \\
\hline Arrival & $326.9(P=30 / 31)$ & n.a. $(P=0 / 25)^{*}$ & n.a. $(P=0 / 50)^{*}$ & n.a. $(P=0 / 30)^{*}$ \\
\hline End & $385.6(P=26 / 26)$ & $503.3(P=23 / 23)$ & $539.0(P=44 / 44)$ & n.a. $(P=0 / 30)^{*}$ \\
\hline \multicolumn{5}{|l|}{ PCV2 } \\
\hline Arrival & $10887.2(P=29 / 31)$ & $159.3(P=25 / 25)$ & $267.0(P=49 / 50)$ & $906.0(P=30 / 30)$ \\
\hline End & $177720.6(P=25 / 26)$ & $54697.9(P=23 / 23)$ & $13704.7(P=41 / 44)$ & $31.9(P=25 / 30)$ \\
\hline
\end{tabular}

${ }^{*}$ n.a.: not applicable, because all titres were 0 .

TABle 5: Position of base differences identified in the full PCV2 genomes isolated from pigs from containers A and B.

\begin{tabular}{lcccc}
\hline Base position & 350 & 1399 & 1405 & 1528 \\
\hline Pig 5 container A & G & G & C & C \\
Pig 31 container A & G & G & C & C \\
Pig 2 container A & A & T & T & T \\
Pig 9 container A & A & T & T & T \\
Pig 18 container A & A & T & T & T \\
Pig 28 container A & A & T & T & T \\
Pig 206 container B & A & T & T & T \\
Pig 217 container B & A & T & T & T \\
\hline
\end{tabular}

\section{Discussion}

To our knowledge this is the first controlled study that documents that PMWS can be induced in pigs from PMWSfree herds exclusively by receiving air from a unit harbouring pigs with clinical PMWS. Previously, we have shown that PMWS can be induced in healthy pigs from PMWS-free herds by direct or indirect contact with pigs from PMWSaffected herds [20] and others have induced PMWS after mingling of pigs inoculated with PCV2 and naïve pigs under experimental conditions $[13,14,27]$. None of the pigs from PMWS-free herds developed clinical signs of PMWS after transportation alone. Therefore, it seems unlikely that stress due to transportation induced PMWS by itself. The fact that the pigs from the PMWS-free herds in units $\mathrm{A}$ and $\mathrm{B}$ but not in $\mathrm{C}$ had a marked increase in PCV2 titer in serum and an increase in antibodies against PCV2 that coincided with the subsequent development of clinical signs typical of PMWS further supported the view that the pigs developed PMWS.

The PMWS diagnosis could not be confirmed by laboratory investigations in a proportion of the pigs with severe clinical signs of PMWS. This has previously been reported from field cases and probably represents end-stage pigs in which the virus level in tissues is low because of massive destruction of cells [7].
The pigs from the PMWS-free herd developed clinical signs of PMWS two weeks after arrival and mixing if they had direct contact with the PMWS-positive pigs (unit A) whereas the pigs in unit $\mathrm{B}$ which had no direct contact with the PMWS pigs developed clinical signs of PMWS three weeks after the start of the study. Previous studies with experimental PCV2 infection in naïve pigs have shown that the transmission of PCV2 is influenced by the contact structure and that direct contact between pigs was more efficient in transmission compared to indirect contact $(10 \mathrm{~cm}$ distance) [13]. Furthermore, the same authors found that the mean disease generation time was 18.4 days following contact with a diseased pig. Accordingly, we have previously shown that disease was induced in PMWS-free pigs 3-4 weeks following mingling with pigs from herds with clinical signs of PMWS and that close contact was more efficient compared to indirect contact [20]. Thus, the finding that the pigs in the present study developed clinical signs of PMWS 2-3 weeks after arrival indicates that the disease generation times in PCV2-positive pigs are comparable to the situation in naïve pigs and that airborne transmission does not delay development of clinical signs.

The viral DNA sequences found in two of the pigs that developed PMWS in unit B were identical and also identical to one of the isolates found in PMWS-affected pigs at arrival. It was not possible to sequence the PCV2 virus from the PMWS-free herd at arrival; however the sequencing results support that the PCV2 virus was transmitted from the PMWS-affected pigs to the pigs from the PMWS-free herds. This is in accordance with our finding in an earlier study on transmission of PMWS by direct and indirect contact [15].

The low levels of antibodies to PRRSV Type I (EU) in the pigs from the PRRSV Type II (US) positive herd probably reflect cross-reaction to PRRSV Type II and the low levels in the remaining pigs probably reflect residue of maternal antibodies. The increase in levels towards PRRSV Type I encountered in some of the pigs at termination of the study probably reflects cross-reaction to PRRSV Type II since these levels were only detected in pigs with very high levels of 
antibodies against PRRSV US. These results strongly indicate that pigs from the PMWS-free herd in units A and B were infected by PRRSV US during the study period and that the source was pigs from the PMWS-affected herd. PRRSV is a well-known infectious trigger of clinical PMWS and vice versa [28-32] and we have previously shown that PRRSV can be transmitted by air [33]. Thus, the finding that most of the pigs that developed PMWS had increased antibody titers against PRRSV Type II suggested that this virus contributed to the clinical manifestation seen in study II. The clinical signs were, however, more in accordance with typical findings in PMWS-affected pigs rather than what is typically seen in pigs acutely infected with PRRSV [29]. The pathological findings at necropsies indeed confirmed that the pigs had developed PMWS. In addition to the role of PRRSV, factors such as differences in PCV2 strain, the dose of PCV2 virus excreted by the "donor" pigs, or even transmission of other unidentified infectious agents from the PMWS-affected pigs to the PMWS-free pigs may have played a role. In conclusion, the present study showed that PMWS can be induced in pigs from a PMWS-free herd by airborne contact with pigs from a PMWS-affected herd. This finding may have implications on the way PMWS is handled in herds.

\section{Acknowledgments}

Financial support to this study was given by EU (Contract no. 513928) and the Research Council for Food, Fisheries and Agri Business (Grant no. 23-04-0242).

\section{References}

[1] J. C. Harding and E. Clark, "Recognizing and diagnosing postweaning multisystemic wasting syndrome (PMWS)," Swine Health and Production, vol. 5, pp. 201-203, 1997.

[2] G. M. Allan and J. A. Ellis, "Porcine circoviruses: a review," Journal of Veterinary Diagnostic Investigation, vol. 12, no. 1, pp. 3-14, 2000.

[3] E. O. Nielsen, C. Enøe, S. E. Jorsal et al., "Postweaning multisystemic wasting syndrome in Danish pig herds: productivity, clinical signs and pathology," Veterinary Record, vol. 162, no. 16, pp. 505-508, 2008.

[4] A. S. Ladekjær-Mikkelsen, J. Nielsen, T. Stadejek et al., "Reproduction of postweaning multisystemic wasting syndrome (PMWS) in immunostimulated and non-immunostimulated 3-week-old piglets experimentally infected with porcine circovirus type 2 (PCV2)," Veterinary Microbiology, vol. 89, no. 2-3, pp. 97-114, 2002.

[5] G. M. Allan, F. McNeilly, S. Kennedy et al., "Isolation of porcine circovirus-like viruses from pigs with a wasting disease in the USA and Europe," Journal of Veterinary Diagnostic Investigation, vol. 10, no. 1, pp. 3-10, 1998.

[6] J. Ellis, S. Krakowka, M. Lairmore et al., "Reproduction of lesions of postweaning multisystemic wasting syndrome in gnotobiotic piglets," Journal of Veterinary Diagnostic Investigation, vol. 11, no. 1, pp. 3-14, 1999.

[7] J. Segalés, C. Rosell, and M. Domingo, "Pathological findings associated with naturally acquired porcine circovirus type 2 associated disease," Veterinary Microbiology, vol. 98, no. 2, pp. 137-149, 2004.
[8] G. M. Allan, F. Mc Neilly, B. M. Meehan et al., "Isolation and characterisation of circoviruses from pigs with wasting syndromes in Spain, Denmark and Northern Ireland," Veterinary Microbiology, vol. 66, no. 2, pp. 115-123, 1999.

[9] R. Magar, R. Larochelle, S. Thibault, and L. Lamontagne, "Experimental transmission of porcine circovirus type 2 (PCV2) in weaned pigs: a sequential study," Journal of Comparative Pathology, vol. 123, no. 4, pp. 258-269, 2000.

[10] N. Rose, T. Opriessnig, B. Grasland, and A. Jestin, "Epidemiology and transmission of porcine circovirus type 2 (PCV2)," Virus Research, vol. 164, no. 1-2, pp. 78-89, 2012.

[11] K. H. West, J. M. Bystrom, C. Wojnarowicz et al., "Myocarditis and abortion associated with intrauterine infection of sows with porcine circovirus," Journal of Veterinary Diagnostic Investigation, vol. 11, no. 6, pp. 530-532, 1999.

[12] S. López-Soria, J. Segalés, N. Rose et al., "An exploratory study on risk factors for postweaning multisystemic wasting syndrome (PMWS) in Spain," Preventive Veterinary Medicine, vol. 69, no. 1-2, pp. 97-107, 2005.

[13] M. Andraud, B. Grasland, B. Durand et al., "Quantification of porcine circovirus type 2 (PCV-2) within- and between-pen transmission in pigs," Veterinary Research, vol. 39, no. 5, article 43, 2008.

[14] M. Andraud, B. Grasland, B. Durand et al., "Modelling the time-dependent transmission rate for porcine circovirus type 2 (PCV2) in pigs using data from serial transmission experiments," Journal of the Royal Society Interface, vol. 6, no. 30, pp. 39-50, 2009.

[15] K. Dupont, C. K. Hjulsager, C. S. Kristensen, P. Baekbo, and L. E. Larsen, "Transmission of different variants of PCV2 and viral dynamics in a research facility with pigs mingled from PMWSaffected herds and non-affected herds," Veterinary Microbiology, vol. 139, no. 3-4, pp. 219-226, 2009.

[16] P. Jaros, L. McIntyre, R. S. Morris, A. C. Johnstone, O. Garkavenko, and E. J. Neumann, "Experimental evidence that an agent other than PCV2 is a necessary cause of PMWS," in Proceedings of the 19th International Pig Veterinary Society Congress Proceedings (IPVS '06), Abstract no. 168, Copenhagen, Denmark, 2006.

[17] H. Vigre, P. Bækbo, S. E. Jorsal et al., "Spatial and temporal patterns of pig herds diagnosed with Postweaning Multisystemic Wasting Syndrome (PMWS) during the first two years of its occurrence in Denmark," Veterinary Microbiology, vol. 110, no. 1-2, pp. 17-26, 2005.

[18] K. A. Woodbine, G. F. Medley, J. Slevin et al., "Spatiotemporal patterns and risks of herd breakdowns in pigs with postweaning multisystemic wasting syndrome," Veterinary Record, vol. 160, no. 22, pp. 751-762, 2007.

[19] P. Wallgren, K. Belák, C. J. Ehlorsson et al., "Postweaning Multisystemic Wasting Syndrome (PMWS) in Sweden from an exotic to an endemic disease," Veterinary Quarterly, vol. 29, no. 4, pp. 122-137, 2007.

[20] C. S. Kristensen, P. Bækbo, V. Bille-Hansen et al., "Induction of porcine post-weaning multisystemic wasting syndrome (PMWS) in pigs from PMWS unaffected herds following mingling with pigs from PMWS-affected herds," Veterinary Microbiology, vol. 138, no. 3-4, pp. 244-250, 2009.

[21] D. Verreault, V. Létourneau, L. Gendron, D. Massé, C. A. Gagnon, and C. Duchaine, "Airborne porcine circovirus in Canadian swine confinement buildings," Veterinary Microbiology, vol. 141, no. 3-4, pp. 224-230, 2010. 
[22] T. K. Jensen, H. Vigre, B. Svensmark, and V. Bille-Hansen, "Distinction between porcine circovirus type 2 enteritis and porcine proliferative enteropathy caused by Lawsonia intracellularis," Journal of Comparative Pathology, vol. 135, no. 4, pp. 176-182, 2006.

[23] A. Bøtner, J. Nielsen, and V. Bille-Hansen, "Isolation of porcine reproductive and respiratory syndrome (PRRS) virus in a Danish swine herd and experimental infection of pregnant gilts with the virus," Veterinary Microbiology, vol. 40, no. 3-4, pp. 351360, 1994.

[24] K. J. Sørensen, B. Strandbygaard, A. Bøtner, E. S. Madsen, J. Nielsen, and P. Have, "Blocking ELISA's for the distinction between antibodies against European and American strains of porcine reproductive and respiratory syndrome virus," Veterinary Microbiology, vol. 60, no. 2-4, pp. 169-177, 1998.

[25] C. K. Hjulsager, L. Grau-Roma, M. Sibila, C. Enøe, L. Larsen, and J. Segalés, "Inter-laboratory and inter-assay comparison on two real-time PCR techniques for quantification of PCV2 nucleic acid extracted from field samples," Veterinary Microbiology, vol. 133, no. 1-2, pp. 172-178, 2009.

[26] K. Dupont, E. O. Nielsen, P. Bækbo, and L. E. Larsen, "Genomic analysis of PCV2 isolates from Danish archives and a current PMWS case-control study supports a shift in genotypes with time," Veterinary Microbiology, vol. 128, no. 1-2, pp. 56-64, 2008.

[27] Y. Okuda, M. Ono, S. Yazawa, and I. Shibata, "Experimental reproduction of postweaning multisystemic wasting syndrome in cesarean-derived, colostrum-deprived piglets inoculated with porcine circovirus type 2 (PCV2): investigation of quantitative PCV2 distribution and antibody responses," Journal of Veterinary Diagnostic Investigation, vol. 15, no. 2, pp. 107-114, 2003.

[28] J. A. Ellis, A. Bratanich, E. G. Clark et al., "Coinfection by porcine circoviruses and porcine parvovirus in pigs with naturally acquired postweaning multisystemic wasting syndrome," Journal of Veterinary Diagnostic Investigation, vol. 12, no. 1, pp. 21-27, 2000.

[29] P. A. Harms, S. D. Sorden, P. G. Halbur et al., "Experimental reproduction of severe disease in $\mathrm{CD} / \mathrm{CD}$ pigs concurrently infected with type 2 porcine circovirus and porcine reproductive and respiratory syndrome virus," Veterinary Pathology, vol. 38, no. 5, pp. 528-539, 2001.

[30] R. M. Pogranichniy, K. J. Yoon, P. A. Harms, S. D. Sorden, and M. Daniels, "Case-control study on the association of porcine circovirus type 2 and other swine viral pathogens with postweaning multisystemic wasting syndrome," Journal of Veterinary Diagnostic Investigation, vol. 14, no. 6, pp. 449-456, 2002.

[31] A. Rovira, M. Balasch, J. Segalés et al., "Experimental inoculation of conventional pigs with porcine reproductive and respiratory syndrome virus and porcine circovirus 2," Journal of Virology, vol. 76, no. 7, pp. 3232-3239, 2002.

[32] N. Rose, E. Eveno, B. Grasland et al., "Individual risk factors for Post-weaning Multisystemic Wasting Syndrome (PMWS) in pigs: a hierarchical Bayesian survival analysis," Preventive Veterinary Medicine, vol. 90, no. 3-4, pp. 168-179, 2009.

[33] C. S. Kristensen, A. Bøtner, H. Takai, J. P. Nielsen, and S. E. Jorsal, "Experimental airborne transmission of PRRS virus," Veterinary Microbiology, vol. 99, no. 3-4, pp. 197-202, 2004. 


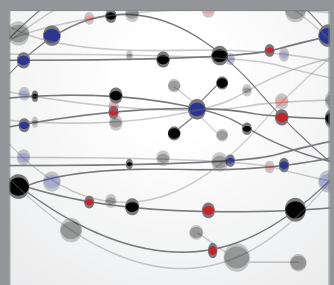

The Scientific World Journal
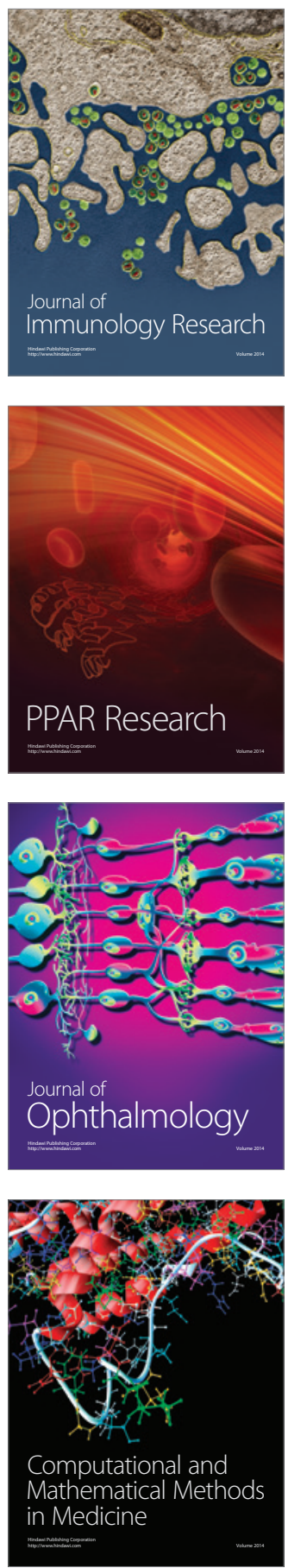

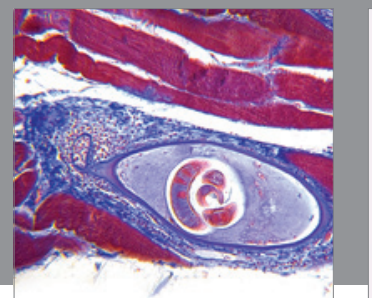

Gastroenterology

Research and Practice
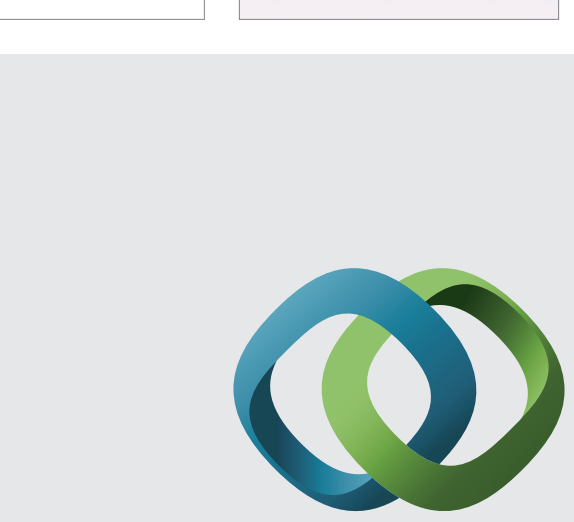

\section{Hindawi}

Submit your manuscripts at

http://www.hindawi.com
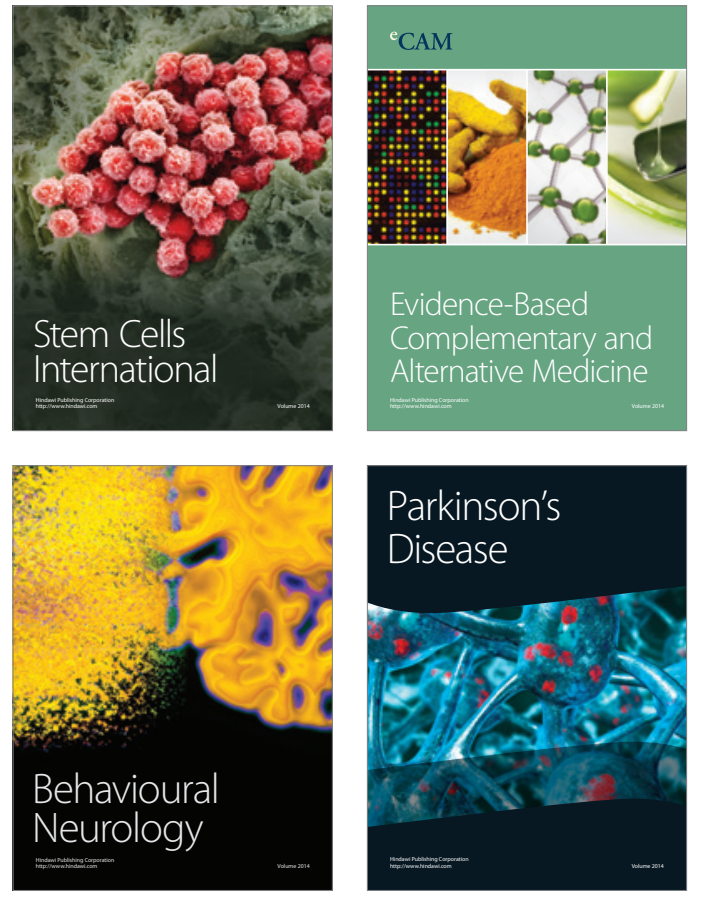
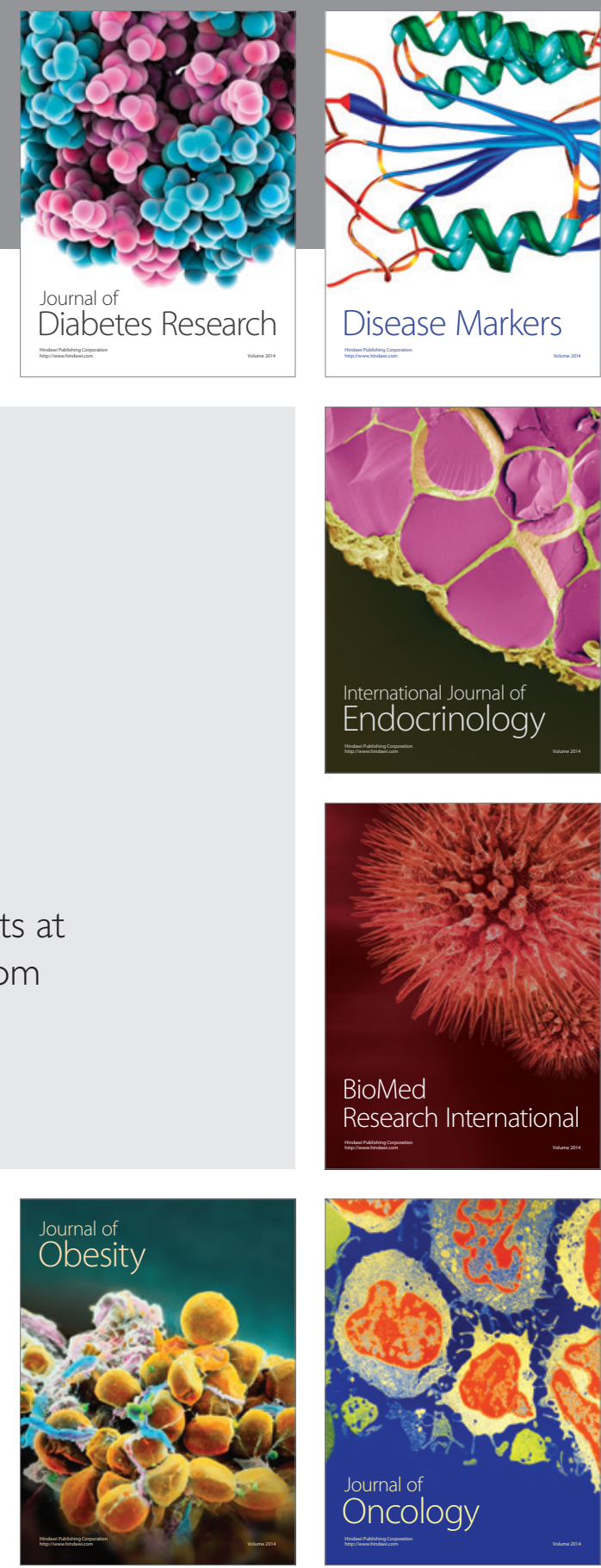

Disease Markers
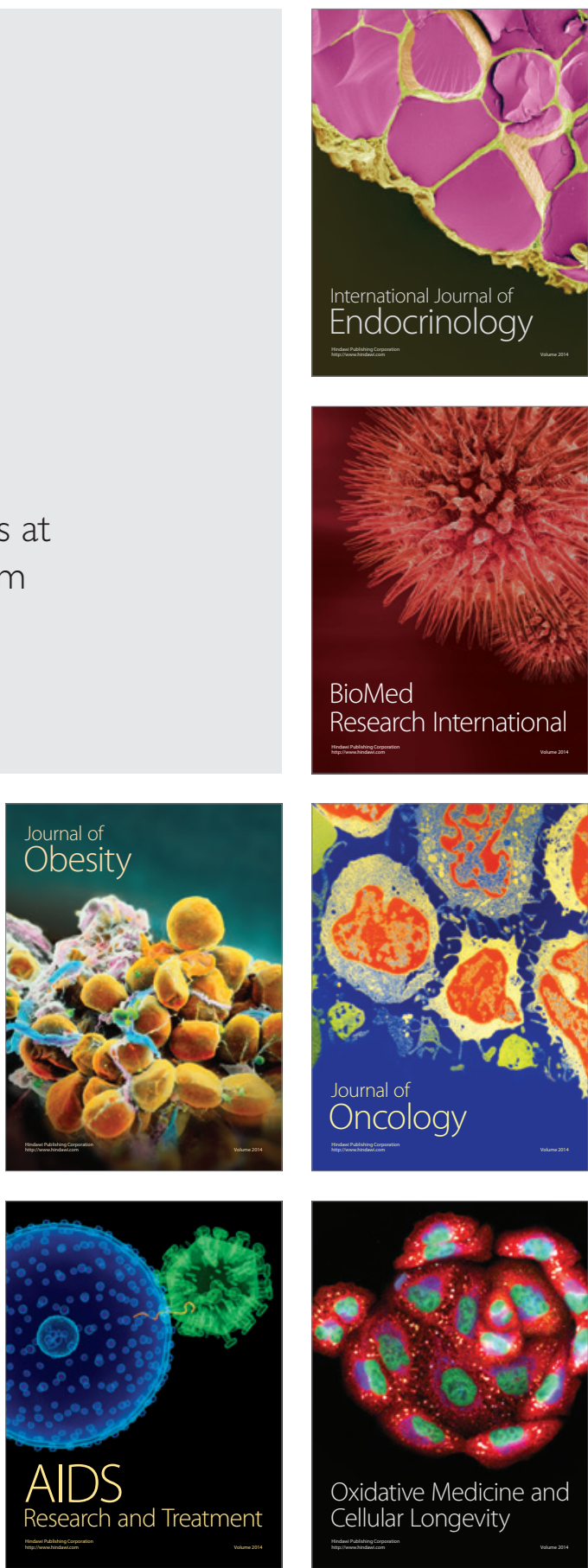